\title{
Phase Transitions in Classical Heisenberg Ferromagnets with Arbitrary Parameter of Anisotropy
}

\author{
V. A. Malyshev \\ Moscow State University, Moscow, USSR
}

Received September 8, 1974

\begin{abstract}
The existence of a phase transition of the first kind is proved for anisotropic classical Heisenberg ferromagnet in two or more dimensions and with arbitrary parameter of anisotropy $\alpha,|\alpha|<1$; a similar fact is proved for much more general lattice spin systems.
\end{abstract}

\section{Introduction}

Bortz and Griffiths proved lately (see [1]) the existence of a phase transition for sufficiently low temperatures in anisotropic classical Heisenberg ferromagnets with small parameter of anisotropy $\alpha(|\alpha|$ $<0.0298$ and $|\alpha|<0.0198$ for a square lattice and simple cubic lattice, respectively). Here we prove the similar result for any $\alpha,|\alpha|<1$. It is the known Fisher's hypothesis (see [2]).

Theorem 1 of our paper contains essentially more general conditions for the existence of a phase transition of the first kind in lattice spin systems with continuous spin space.

The main difference between our method and the method of [1] is the following: in [1] the sharp "border" is constructed and we construct a spread gradually altering "border" (Bloch wall).

It is interesting to compare our result with the result of Mermin and Wagner (see [3]) about the impossibility of phase transitions of the sort considered here for the square lattice and for $|\alpha|=1$.

\section{Formulation of the Main Result}

Let $\mathbb{T}$ be an abelian group $\mathbb{Z}^{v}, v \geqq 2$, where $\mathbb{Z}$ is a group of integers. Let $S$ be a compact separable metric space with finite nonnegative measure $\mu$ defined on Borel subsets of $S$. Assume to be given a real measurable function $U\left(s_{1}, s_{2}\right)=U\left(s_{2}, s_{1}\right)$ on $S \times S$ which is bounded from below on $S \times S$.

We shall consider Gibbsian random fields on a lattice $\mathbb{T}$ with values in $S$ for any $t \in T$ (see $[4,6]$ ). For simplifying notations we shall discuss 
in the sequel only the case $v=2$ bearing in mind that extension for the case $v>2$ is trivial.

Let us denote

$$
\Delta=\{t: t \in \mathbb{T},|t|=1\},
$$

where $|t|$ is the length of the vector $t$.

It is known [4], that there exists at least one limit Gibbsian distribution of a random field such that for any finite set

$$
\mathbb{V}=\left\{t_{1}, \ldots, t_{v}\right\} \subset \mathbb{T}
$$

( $v$ is a number of elements in $\mathbb{V}$ ) and for any given configuration $s(t)$ outside $\mathbb{V}$ (i.e. function on $\mathbb{T}-\mathbb{V}$ with values in $S$ ) conditional probability distributions are given with probability one by

$$
q_{v}\left(s_{1}, \ldots, s_{v} / s(t)\right)=\frac{\exp \left\{-\beta U_{\mathbb{V}}\left(s_{1}, \ldots, s_{v} / s(t)\right)\right\}}{\int \exp \left\{-\beta U_{\mathbb{V}}\left(s_{1}, \ldots, s_{v} / s(t)\right) d \mu\left(s_{1}\right) \ldots d \mu\left(s_{v}\right)\right.}
$$

where

$$
U_{\mathbb{V}}\left(s_{1}, \ldots, s_{v} / s(t)\right)=\sum_{t_{2}-t_{j} \in \Delta} U\left(s_{i}, s_{j}\right) / 2+\sum_{i=1}^{v} \sum_{t \in \mathbb{V}} U\left(s_{i}, s(t)\right) .
$$

A measurable one-to-one transformation $h$ of a measure space $\left(\mathscr{X}_{1}, \Sigma_{1}, \mu_{1}\right)$ to a measure space $\left(\mathscr{X}_{2}, \Sigma_{2}, \mu_{2}\right)$ we shall call admissible if measure $\mu_{1} h^{-1}$ on $\mathscr{X}_{2}$ is absolutely continuous with respect to $\mu_{2}$ and the corresponding Radon-Nykodim derivative satisfies the following inequality

$$
0<c_{1} \leqq \frac{d\left(\mu_{1} \cdot h^{-1}\right)}{d\left(\mu_{2}\right)} \leqq c_{2}<\infty
$$

almost everywhere on $\left(\mathscr{X}_{2}, \Sigma_{2}, \mu_{2}\right)$ for some $c_{1}$ and $c_{2}$.

Further the following conditions for a function $U\left(s_{1}, s_{2}\right)$ will be fundamental.

1. $U\left(s_{1}, s_{2}\right)$ is finite and continuous on $S \times S$ and takes its absolute minimum exactly in two points $\left(s_{0}, s_{0}\right)$ and $\left(s_{0}^{\prime}, s_{0}^{\prime}\right)$ on the diagonal of $S \times S$.

2. There exists a one-to-one transformation $g: S \rightarrow S$ which conserves measure $\mu$ and is such that $g s_{0}=s_{0}^{\prime}$ and $g s_{0}^{\prime}=s_{0}, U\left(s_{1}, s_{2}\right)$ $=U\left(g s_{1}, g s_{2}\right)$.

3. There exist two neighbourhoods $\mathrm{O}_{1}$ and $\mathrm{O}_{2}, \mathrm{O}_{1} \subset \mathrm{CO}_{2}$, of the point $s_{0}$, such that $\mu\left(O_{1}\right)>0, O_{2} \bigcap g O_{2}=\emptyset$, and for some $\varepsilon>0$

$$
U\left(s_{1}, s_{2}\right)<U\left(s_{1}^{\prime}, s_{2}^{\prime}\right)-\varepsilon
$$

for any $s_{1}, s_{2} \in O_{1}$ and any $s_{1}^{\prime}, s_{2}^{\prime}$ such that they cannot simultaneously belong neither $\mathrm{O}_{2}$ nor $g \mathrm{O}_{2}$. 
4. If $\mu\left(O_{2}-O_{1}\right)>0$ then there exists an admissible transformation $\chi$ of measure space $O_{2}-O_{1}$ to some Borel $A_{0} \subset O_{1}$ with induced measure $\mu$ such that

$$
U\left(s_{1}, \chi s_{2}\right)<U\left(s_{1}, s_{2}\right)-\varepsilon
$$

for any $s_{1} \in O_{1}, s_{2} \in O_{2}-O_{1}$, and

$$
U\left(\chi s_{3}, \chi s_{2}\right)<U\left(s_{3}, s_{2}\right)-\varepsilon
$$

for any $s_{2}, s_{3} \in O_{2}-O_{1}$.

5. There exists a partition of the set $\mathrm{S}-\left(\mathrm{O}_{2} \cup \mathrm{gO} \mathrm{O}_{2}\right)$ with finite number of nonintersecting subsets $F_{i}, i=1, \ldots, k$,

$$
\bigcup_{i=1}^{k} F_{i}=S-\left(O_{2} \bigcup g O_{2}\right), \mu\left(F_{i}\right)>0
$$

and admissible transformations $f_{i}: F_{i} \rightarrow A_{i} \subset O_{1}, \mu\left(A_{i}\right)>0$ (on $F_{i}$ and $A_{i}$ the induced measure $\mu$ is given).

Let us denote

$$
b=\min \left\{\frac{d\left(\mu \circ f_{i}\right)}{d \mu}, \frac{d(\mu \circ \chi)}{d \mu}\right\}>0 .
$$

We formulate now the main result of this paper.

Theorem 1. If a function $U\left(s_{1}, s_{2}\right)$ satisfies the Conditions $1-5$ then for sufficiently small temperature $T=1 / \beta$ there exist at least two different Gibbsian limit distributions with given conditional probability distributions (1).

Remark 1. As it will be evident from the proof given below Theorem 1 admits some generalisations: for example, for finite particle (translation invariant or periodic) interaction $U(X)$, where $X \subset S$ - an arbitrary finite set. Roughly speaking it is necessary then to demand that conditions similar to $1-5$ hold for all $U(X)$ simultaneously.

Remark 2. The number $\beta$ for which nonuniqueness already takes place is estimated in the proof. This estimate depends strongly on $1-|\alpha|$. For obtaining better estimates it is necessary to choose optimal $O_{1}$ and $\mathrm{O}_{2}$. Here we do not discuss this question.

\section{Proof of Theorem 1}

Let $A$ be an arbitrary measurable subset of $S$ with positive measure $\mu$.

Further we shall consider the set $\mathbb{V}$ of all sites of lattice $\mathbb{T}$ within a square with centre in the origin and which we shall assume sufficiently large.

A site $t \in \mathbb{T}$ is called an $A$-site of a configuration $s(t)$ if $s(t) \in A$. We shall consider only such configurations all sites of which outside $\mathbb{V}$ are $O_{1}$-sites. 
Let us call sites $t_{1}, t_{2} \in \mathbb{T}$ interacting if $t_{1}-t_{2} \in \Delta$. Sets $\mathbb{T}^{\prime} \subset \mathbb{T}$ and $\mathbb{T}^{\prime \prime} \subset \mathbb{T}$ we call interacting if there exist at least two interacting sites $t_{1} \in \mathbb{T}^{\prime}$ and $t_{2} \in \mathbb{T}^{\prime \prime}$. We shall call a sequence of sites $t^{\prime}=t_{1}, \ldots, t_{n}=t^{\prime \prime}$ such that $\left|t_{i}-t_{i+1}\right| \leqq d$ for $i=1, \ldots, n-1$ a $d$-path connecting sites $t^{\prime}$ and $t^{\prime \prime}$. A subset $\mathbb{T}^{\prime} \subset \mathbb{T}$ is called $d$-connected if its any two sites can be connected with a $d$-path.

We denote $A=S-O_{1}, B=g O_{1}$.

Let us consider any site $t_{0} \in \mathbb{V}$. If we shall be able to prove that a probability for $t_{0}$ to be an $A$-site is less than $1 / 2$, then in a standard way with the use of the "symmetry" $g$ Theorem 1 follows. The proof given below uses Peierls argument and is similar in some places to known proofs (e.g., [1] and [5]). Essential complications arise due to specific character of continuous spins. That is why we pay our attention only on new elements in proof without repeating as far as possible well known arguments.

Let $t_{0}$ be $A$-site and $R_{A}$ - maximal 1 -connected component of $A$-sites which contains $t_{0}$.

A set $\mathbb{T}-R_{A}$ can be decomposed into several 1-connected components $R_{0}, R_{1}, \ldots, R_{q}$. The component which contains $\mathbb{T}-\mathbb{V}$ we shall call outer and denote it $R_{0}$.

We shall now define inductively a set $R_{A}(B)$-an outer $B$-border of $R_{A}$. to $R_{A}$ :

By definition $R_{A}(B)$ consists of and only of the following sites belonging

1. the sites which interact with $R_{0}$;

2. sites which are not $B$-sites and which interact with at least one site of $R_{A}(B)$ defined earlier;

3. $B$-sites interacting with at least one defined earlier site of $R_{A}(B)$ which is not a $B$-site;

4. the sites interacting with those $R_{i}$ which contain at least one site interacting with at least one site of $R_{A}(B)$ defined earlier.

The union of $R_{A} R_{A}(B)$ and those $R_{i}$ which do not interact with $R_{A}(B)$ we denote $R_{A}^{0}$.

The union of $R_{A}-R_{A}(B)$ and those $R_{i}$ which do not interact with $R_{A}(B)$ we denote $R_{A}^{0}$.

Lemma 1. The set $R_{A}(B)$ is $\sqrt{2}$-connected.

Proof. For any 1-connected set $\mathbb{C} \subset \mathbb{V}$ the set of sites belonging to outer component of $\mathbb{T}-\mathbb{C}$ and interacting with $\mathbb{C}$ is $\sqrt{2}$-connected. This fact is evident from geometrical considerations after constructing unit squares with centres in sites of $\mathbb{T}$.

From this the proof of Lemma 1 is completed by induction.

Lemma 2. The number of $\sqrt{2}$-connected sets containing the fixed site $t$ and consisting of exactly $l$ sites does not exceed $8^{l-1}$. 
Proof. We shall numerate sites of such set $L$. Let us put $t_{1}=t$. On the first step all sites which are located at the distance not more than $\sqrt{2}$ from $t_{1}$ and belong to $L$ we shall numerate after $t_{1}$ in some fixed (e.g., lexicographical) order.

Let $m=m(k) \geqq k$ sites be numerated after $k$ steps. One the $(k+1)$ step we take site $t_{k}$ and numerate with numbers $m+1, m+2$ and so on the sites of $L$ which we have not numerated earlier and located at the distance not more than $\sqrt{2}$ from $t_{k}$ (the number of such sites does not exceed 8). The number of possible sequences of sites increases on each step not more than in 8 times. Consequently, the number of all possible sequences does not exceed $8^{l-1}$. From this Lemma 2 follows.

We get in a standard way from the Lemma 2 that the number of possible sets $R_{A}(B)$ consisting of exactly $l$ sites does not exceed $c l^{2} 8^{l}$ for some constant $c$.

We define now a transformation $G$ of a space of configurations into itself

$$
G s(t)=\left\{\begin{aligned}
(g s)(t), & t \in R_{A}^{0} \\
s(t), & t \in \mathbb{T}-\left(R_{A} \bigcup R_{A}^{0}\right) \\
(f s)(t), & t \in R_{A}-R_{A}^{0}=R_{A}(B)
\end{aligned}\right.
$$

where $f$ is defined in the following way:

$$
f s= \begin{cases}s, & s \in O_{1} \\ \chi s, & s \in O_{2}-O_{1} \\ f_{i} s, & s \in F_{i} \\ g s, & s \in g O_{1} \\ \chi g s, & s \in g\left(O_{2}-O_{1}\right) .\end{cases}
$$

Let us decompose the set of all configurations into several subsets (clusters) in the following way. We consider a partition $\mathfrak{R}$ of $S$ into subsets $F_{1}, \ldots, F_{k}, g O_{1}, g\left(O_{2}-O_{1}\right), O_{2}-O_{1}, O_{1}$. Two configuration $s_{1}(t)$ and $s_{2}(t)$ belong to the same cluster iff for any $t \in R_{A}(B)$ the following is true: $s_{1}(t)$ and $s_{2}(t)$ belong to the same element of partition $\mathfrak{R}$.

The number of possible clusters does not exceed $(k+3)^{l}$.

Let us consider any such cluster $L$, partition function $Z_{L}$ over all configurations of this cluster and partition function $Z_{\mathrm{GL}}$ over all configurations of the set GL (with fixed configuration of $O_{1}$-sites outside $\mathbb{V}$ ).

It is easy to verify that for any two configurations $s(t)$ and $G s(t)$ the quantities

and

$$
U_{s}=U\left(s(t), t \in \mathbb{V} / s(t) \in O_{1}, t \bar{\epsilon} \mathbb{V}\right)
$$

$$
U_{G s}=U\left(G s(t), t \in \mathbb{V} / G s(t) \in O_{1}, t \bar{\in} \mathbb{V}\right)
$$


satisfy the following inequality

$$
U_{G s} \leqq U_{s}-\varepsilon \beta l / 2 .
$$

As $g$ is a measure conserving transformation and the rest transformations are admissible then the following inequality takes place

$$
Z_{L} / Z_{\mathrm{GL}} \leqq \exp (-l \varepsilon \beta / 2) / b^{l} \text {. }
$$

Gathering all estimates and considering

$$
\sum_{l} c l^{2} 8^{l}(k+3)^{l} \exp (-l \varepsilon \beta / 2) b^{-l}
$$

we can now prove Theorem 1 in an usual way.

\section{Heisenberg Ferromagnet}

In anisotropic classical Heisenberg ferromagnet $S$ is a unit sphere, i.e. $S=\left\{s=(x, y, z): x^{2}+y^{2}+z^{2}=1\right\}$ and

$$
U\left(s_{1}, s_{2}\right)=-J\left(z_{1} z_{2}+\alpha\left(x_{1} x_{2}+y_{1} y_{2}\right)\right)
$$

for some real $J>0$ and $|\alpha|<1$. We can put further $J=1$.

The transformation $g$ maps a point $(x, y, z)$ onto $(x, y,-z)$, and $\mu$ is a measure on $S$ invariant with respect to euclidean rotations and to the transformation $g$.

Let us put

$$
\begin{aligned}
& O_{1}=\left\{s: z>0, r=\sqrt{x^{2}+y^{2}}<\delta\right\}, \quad \delta>0, \\
& O_{2}=\{s: z>0, r<2 \delta\} .
\end{aligned}
$$

We define a transformation $\chi$ in coordinates $r$ and $\varphi(x=r \cos \varphi, y=r \sin \varphi)$ in the following way:

$$
\chi(\varphi)=\varphi, \chi(r)=\delta-(2 \delta-r)(1-|\alpha|) / 2 .
$$

We shall verify the Conditions $1-5$ for $U\left(s_{1}, s_{2}\right)$ putting

$$
\varepsilon=(1-|\alpha|)^{2} \delta^{2} / 5 \text {. }
$$

1. If $|\alpha|<1$ then $z_{1} z_{2}+\alpha\left(x_{1} x_{2}+y_{1} y_{2}\right)$ has its maximum exactly in two points $\left(s_{0}, s_{0}\right)$ and $\left(s_{0}^{\prime}, s_{0}^{\prime}\right)$ on the diagonal of $S \times S$ where $s_{0}=(0,0,1)$, $s_{0}^{\prime}=(0,0,-1)$. This can be verified in an obvious way.

2. Condition 2 is evident.

3. Further we assume $\delta$ to be sufficiently small. That is why we should only prove that

$$
U\left(s_{1}, s_{2}\right)<U\left(s_{1}^{\prime}, s_{2}^{\prime}\right)-\varepsilon
$$

for any $s_{1}, s_{2} \in O_{1}, s_{1}^{\prime} \in S-\left(O_{2} \bigcup g O_{2}\right), s_{2}^{\prime} \in S$. 
We have

$$
\begin{aligned}
a=\sup _{s_{1}, s_{2} \in O_{1}}\left(-z_{1} z_{2}-\alpha\left(x_{1} x_{2}+y_{1} y_{2}\right)\right) & =\inf _{s_{1}, s_{2} \in O_{1}}\left(z_{1} z_{2}+\alpha\left(x_{1} x_{2}+y_{1} y_{2}\right)\right) \\
& =-\inf _{s_{2} \in O_{1}}\left(\sqrt{1-\delta^{2}} z_{2}+\alpha \delta x_{2}\right)
\end{aligned}
$$

as $U\left(s_{1}, s_{2}\right)$ is invariant with respect to rotations around $z$-axis and, consequently, we can put $s_{1}$ equal to $\left(\delta, 0, \sqrt{1-\delta^{2}}\right)$.

Consequently

$$
a=-\left(1-\delta^{2}-|\alpha| \delta^{2}\right)=-1+\delta^{2}(1+|\alpha|)
$$

Similarly, putting $s_{2}^{\prime}=\left(x_{2}^{\prime}, 0, z_{2}^{\prime}\right)$, we have

$$
\begin{aligned}
b & =\inf _{\substack{s_{1}^{\prime} \in S-\left(O_{2} \cup g o_{2}\right) \\
s_{2}^{\prime} \in S}}\left(-\left(z_{1}^{\prime} z_{2}^{\prime}+x_{1}^{\prime} x_{2}^{\prime}\right)\right)=\underset{\left|z_{1}^{\prime}\right| \leqq \sqrt{1-4 \delta^{2}}}{-\sup _{\left|z_{2}^{\prime}\right| \leqq 1}}\left(z_{1}^{\prime} z_{2}^{\prime}+\alpha x_{1}^{\prime} x_{2}^{\prime}\right) \\
& =-\max \left(|\alpha|, \sqrt{1-4 \delta^{2}}\right)=-\sqrt{1-4 \delta^{2}}=-1+2 \delta^{2}+o\left(\delta^{2}\right) .
\end{aligned}
$$

It follows

$$
\begin{aligned}
a-b & =-\delta^{2}(2-(1-|\alpha|))+o\left(\delta^{2}\right) \\
& =-\delta^{2}(1-|\alpha|)+o\left(\delta^{2}\right) \leqq-\varepsilon .
\end{aligned}
$$

4. We shall prove that

$$
U\left(s_{1}, \chi s_{2}\right)<U\left(s_{1}, s_{2}\right)-\varepsilon
$$

for any $s_{1} \in O_{1}, s_{2} \in O_{2}-O_{1}$.

Let $s_{1}=\left(r_{1}, \varphi_{1}\right), s_{2}=\left(r_{2}, \varphi_{2}\right)$. Then

$$
\begin{aligned}
U\left(s_{1}, s_{2}\right)= & -\sqrt{1-r_{1}^{2}} \sqrt{1-r_{2}^{2}} \\
& -r_{1} r_{2}\left(\cos \varphi_{1} \cos \varphi_{2}+\sin \varphi_{1} \sin \varphi_{2}\right), \\
\frac{\partial U}{\partial r_{2}}= & \frac{\sqrt{1-r_{1}^{2}}}{\sqrt{1-r_{2}^{2}}} r_{2}-\alpha r_{1}\left(\cos \varphi_{1} \cos \varphi_{2}+\sin \varphi_{1} \sin \varphi_{2}\right) \\
\geqq & \frac{\sqrt{1-r_{1}^{2}}}{\sqrt{1-r_{2}^{2}}} r_{2}-|\alpha| r_{1}=r_{2}-|\alpha| r_{1}+O\left(\delta^{2}\right) \\
\geqq & (1-|\alpha|) / 2+O\left(\delta^{2}\right)
\end{aligned}
$$

for

$$
r_{2} \geqq \delta\left(1-\frac{1-|\alpha|}{2}\right), \quad r_{1} \leqq \delta .
$$

Then

as

$$
\begin{gathered}
U\left(s_{1}, s_{2}\right)-U\left(s_{1}, \chi s_{2}\right) \\
\geqq\left(r_{2}-\chi\left(r_{2}\right)\right) \inf \frac{\partial U}{\partial r_{2}} \geqq \frac{1-|\alpha|}{2} \delta \frac{1-|\alpha|}{2} \delta+O\left(\delta^{3}\right) \geqq \varepsilon
\end{gathered}
$$

$$
r_{2}-\chi\left(r_{2}\right) \geqq \frac{1-|\alpha|}{2} \delta .
$$


Similarly for any $s_{1}=\left(r_{1}, \varphi_{1}\right), s_{2}=\left(r_{2}, \varphi_{2}\right) \in O_{2}-O_{1}$ we have

$$
\begin{aligned}
& U\left(s_{1}, s_{2}\right)-U\left(\chi s_{1}, \chi s_{2}\right)=r_{1}^{2} / 2+r_{2}^{2} / 2-\alpha r_{1} r_{2}\left(\cos \varphi_{1} \cos \varphi_{2}+\sin \varphi_{1} \sin \varphi_{2}\right) \\
& -\left(\chi\left(r_{1}\right)\right)^{2} / 2-\left(\chi\left(r_{2}\right)\right)^{2} / 2+\alpha\left(\chi\left(r_{1}\right)\right)\left(\chi\left(r_{2}\right)\right)\left(\cos \varphi_{1} \cos \varphi_{2}+\sin \varphi_{1} \sin \varphi_{2}\right) \\
& +o\left(\delta^{2}\right) \geqq r_{1}^{2} / 2+r_{2}^{2} / 2-|\alpha| r_{1} r_{2}-\left(\chi\left(r_{1}\right)\right)^{2} / 2-\left(\chi\left(r_{2}\right)\right)^{2} / 2+|\alpha|\left(\chi\left(r_{1}\right)\right)\left(\chi\left(r_{2}\right)\right) \\
& +o\left(\delta^{2}\right)=\left(r_{1}-r_{2}\right)^{2} / 2+(1-|\alpha|) r_{1} r_{2}-\left(\chi\left(r_{1}\right)-\chi\left(r_{2}\right)\right)^{2} / 2 \\
& -(1-|\alpha|)\left(\chi\left(r_{1}\right)\right)\left(\chi\left(r_{2}\right)\right)+o\left(\delta^{2}\right) \geqq(1-|\alpha|) \delta^{2}\left(1-|\alpha|-(1-|\alpha|)^{2} / 4\right) \\
& +o\left(\delta^{2}\right) \geqq 3(1-|\alpha|)^{2} \delta^{2} / 4+o\left(\delta^{2}\right) \geqq \varepsilon
\end{aligned}
$$

as

and as

$$
\left|r_{1}-r_{2}\right| \geqq\left|\chi\left(r_{1}\right)-\chi\left(r_{2}\right)\right|
$$

$$
\inf _{s_{1}, s_{2} \in O_{2}-O_{1}}\left(r_{1} r_{2}-\left(\chi\left(r_{1}\right)\right)\left(\chi\left(r_{2}\right)\right)\right)
$$

is reached for $r_{1}=r_{2}=\delta$.

5. Condition 5 is evident.

We have proved thus the following

Corollary 1. Classical Heisenberg ferromagnet with arbitrary parameter of anisotropy $\alpha,|\alpha|<1$, has a nonzero spontaneous magnetization for sufficiently low temperatures.

Remark 3. It follows from Theorem 1 also the existence of a phase transition in several other models, for example, in Vahcks-Larkin model (see [7]). Griffiths result [8], where $S$ is a unit closed interval, also can be deduced from our result.

\section{References}

1. Bortz, A., Griffiths, R.: Commun. math. Phys. 26, 102-108 (1972)

2. Fisher, M.: J. Appl. Phys. 38, 981 (1967)

3. Mermin, N. D., Wagner, H.: Phys. Rev. Letters 17, 1133 (1966)

4. Dobrushin, R. L.: Teoriya Veroyatnostei i ee Primeneniya 15, 469-497 (1970)

5. Berezin, F.A., Sinai, Ya. G.: Truhdy of Moscow Mathem. Soc. 17, 197-212 (1967)

6. Dobrushin, R. L.: Funct. Analiz Pril. 2, No. 4, 31-43 (1968)

7. Stanley, H.E.: Introduction to phase transitions and critical phenomena. Oxford: Clarendon Press 1971

8. Griffiths, R.: J. Math. Phys. 10, 1559-1565 (1969)

Communicated by G. Gallavotti

V. A. Malyshev

Faculty of Mechanics and Mathematics

Chair of Probability

Moscow State University

Leninskie Gory

Moscow V-234, USSR 